\title{
The Potential of Protein Expression Profiles in Categorization Risks for Acute Myeloid Leukemia: A Pilot Study
}

\author{
Amer ALMAIMAN ${ }^{1,2}$, Rasedee ABDULLAH ${ }^{1}$, Ahmad B. bin ABDUL ${ }^{1}$, Zeenathul N. ALLAUDDIN', \\ Ayodele A. ALAIYA ${ }^{2}$, Eltayeb EM. EID ${ }^{3}$, Zakia SHINWARI ${ }^{2}$, Ghada AI JUHANI ${ }^{4}$, Walid RASHEED ${ }^{4}$, \\ Nasir BAKSHI ${ }^{5}$, Tareq OWAIDAH ${ }^{5}$, Syed O. AHMED ${ }^{2}$, Mahmoud ALJURF ${ }^{4}$ \\ ${ }^{1}$ Universiti Putra Malaysia, Institute of Bioscience, Malaysia \\ ${ }^{2}$ King Faisal Specialist Hospital and Research Center, Proteomics Unit \\ Stem Cells \& Tissue Re-Engineering Program, Riyadh, Saudi Arabia \\ ${ }^{3}$ Qassim University, Uniazah College of Pharmacy, Qassim, Saudi Arabia \\ ${ }^{4}$ King Faisal Specialist Hospital and Research Center, Oncology Center, Riyadh, Saudi Arabia \\ ${ }^{5}$ King Faisal Specialist Hospital and Research Center, Department of Pathology \\ and Laboratory Medicine, Riyadh, Saudi Arabia
}

\begin{abstract}
Currently, there are no markers to predict response to acute myeloid leukemia (AML) therapy and patients have to wait for a period of 3-6 months to see treatment response. The study aimed to analyze changes in protein expression in AML cells between different categorization risk groups using proteomics techniques. Six peripheral blood (PB) and six bone marrow (BM) samples at diagnosis and remission times were collected from AML patients. Another Six PB samples were collected from different categories of AML. All samples were analyzed by liquid chromatography tandem mass spectrometry (LC-MS/MS). The levels of proteins in patients with AML were compared at different categorization risk, individual response to treatment and clinical characteristics. Twenty-one and 145 differentially expressed proteins were identified with disease progression and risk categories of AML, respectively. Three (3) proteins were noticeably highly expressed out of the range of others proteins by at least 3-fold difference between diagnosis and remission. Two other of proteins were up regulated by more than 10 folds between risk categories of AML. Furthermore, 4 proteins were found to be expressed in one risk category, but were not detectable in other two risk categories. The study showed that a panel of differentially expressed protein profiles might serve as more objective biomarkers for accurate stratification of different risk categories of AML.
\end{abstract}

Keywords: Acute Myeloid Leukemia, Proteomics, Risk category, Biomarker discovery

\section{ÖZET}

\section{Akut Miyeloid Lösemide Protein Ekspresyon Profillerinin Potansiyeli: Ön Çalışma}

Günümüzde, akut miyeloid lösemide (AML) tedavi yanıtını belirleyen marker yoktur. Hastalar, tedavi yanıını görebilmek için 3-6 ay kadar beklemeleri gerekmektedir. Bu çalışmada farklı risk gruplarında AML hücrelerinin protein ekspresyon değişiklikleri, proteomiks tekniği ile çalışılmıştır. Altı AML hastasının tanı anında ve remisyonda alınan periferik kan (PK) ve kemik iliği (Ki) örnekleri analiz edilmiştir. Diğer risk kategorisinden 6 AML hastasının PK analiz edildi. Örneklerin hepsi liquid chromatography tandem mass spectrometry (LC-MS/MS)'de analiz edildi. Hastaların protein ekspresyonları, farklı risk kategorisine, tedavi yanıtına ve klinik özelliklerine göre analiz edildi. Hastalık tedavi yanıtlarına göre 21, risk gruplarına göre bakıldığında ise 145 farkı protein ekspresyonun olduğu saptandı. Üç proteinin tanı anında ve remsiyonda normal aralıkların çok üzerinde; en az üç kat yüksek olarak eksprese olduğu saptandı. Farklı iki proteinin ise farklı risk gruplarında en az 10 kat faarklı olarak eksprese olduğu görüldü. Ayrıca, 4 protein bir risk grubunda eksprese olur ikeni, diğer iki risk grubunda ekspresyonu saptanacak düzeylerde değildi. Bu çalışma farklı protein ekspresyon panlellerinin, farklı risk sınıflamalarında daha objektif biyomarker olarak kullanılabileceğini göstermiştir.

Anahtar Kelimeler: Akut Miyeloid Lösemi, Protemiks, Risk kategorisi, Biyomarker keşfi 


\section{INTRODUCTION}

Leukemia is a malignant disorder of hematopoietic stem cells (HSCs) that occurs as a result of uncontrolled growth and differentiation as well as self-renewal capacity. In acute myeloid leukemia (AML), HSCs persist in an immature state because their development or differentiation is blocked at the level of progenitor cells. Consequently, leukemic cells (blasts) reproduce and accumulate rapidly. ${ }^{1}$ One of the main goals of proteomics studies in cancer research is to characterize proteins expressed in abnormal cells. Basically, proteomics has the capacity to identify abnormally expressed proteins in malignant cells and the expression of proteins of the metabolic and signaling pathways will help to elucidate the mechanisms underlying the initiation and development of neoplasia. ${ }^{2}$ Advancement technologies in proteomics studies have increased the opportunities for discovering diseases biomarkers, particularly cancers. The detection of sensitive biomarkers present at low concentrations in human plasma of AML patients has a higher value for development of new methods of determining the prognosis of AML. Such biomarkers will complement the more classical diagnostic techniques such as cytogenetic, hematology, and other clinical tests conducted on a routine basis. More importantly, this innovative approach would minimize the use of invasive procedures such as bone marrow biopsies, thereby avoiding associated complications. ${ }^{3,4}$

Survival of AML patients is dependent on strategic therapy. Mortality in these patients is often related to failure in the initiation of chemotherapy and early relapse. ${ }^{5}$ Thus, risk stratification based on prognostic factors is essential to adapt a therapeutic regime for positive clinical outcome. This may be achieved through profiling of differentially expressed plasma peptides and mapping the peptide spectrum specific for the AML subtype. Proteomic profiling is also useful for the clarification of pathogenesis, drug-resistance and prognosis. ${ }^{6} \mathrm{Con}$ sequently, this study was design to analyze changes in protein expression of AML cells between different categorization risk groups using proteomics techniques that could potentially shed insights into leukemic pathophysiology and help in predicting early responses to treatment.

\section{MATERIALS AND METHODS}

\section{Study Subjects}

Written informed consent was obtained from all participants. The Research Advisory Council and the Office of Research Affairs, King Faisal Specialist Hospital and Research Center, Riyadh, Saudi Arabia approved this study. The disease data were compiled and compared against the protein fingerprints derived from the patients' peripheral blood and bone marrow samples.

\section{Sample Collection and Preparation}

Peripheral blood $(5 \mathrm{~mL})$ and bone marrow $(2 \mathrm{~mL})$ were collected from patients with AML in ethylenediamine tetraacetic acid (EDTA) tubes at diagnosis and remission time. Blood samples were subjected to Ficoll-Paque density gradient centrifugation to obtain plasma and mononuclear cells as previously described. ${ }^{5}$ Briefly, fresh balanced salt solution (BSS) was prepared by adding $1 \mathrm{mM}$ phenylmethylsulfonyl fluoride (PMSF) to phosphatebuffered saline (PBS) to a final concentration of $20 \mu \mathrm{M}$ and benzamidine at a final concentration of $830 \mu \mathrm{M}$. The PMSF was dissolved in ethanol while benzamidine was dissolved in distilled water. Equal volumes of EDTA anti-coagulated blood and freshly prepared BSS were added to a $15-\mathrm{mL}$ tube, mixed thoroughly, and gently vortexed. A $22 \mathrm{G}$ syringe needle was inserted through the septum of a bottle containing well-mixed Ficoll-Paque PLUS (GE Healthcare, Pittsburgh, PA, USA), the bottle inverted, and required volume of FicollPaque PLUS withdrawn. A 22G needle and syringe was inserted vertically into the $15-\mathrm{mL}$ test tube containing anti-coagulated blood until it reached the bottom. The Ficoll-Paque PLUS was gently and slowly released to form a layer under the blood sample, while avoiding mixing. The sample then was centrifuged for $30 \mathrm{~min}$ at $318 \times \mathrm{g}$ (Eppendorf, Hamburg, Germany) at room temperature. The upper layer was withdrawn using a clean pasture pipette, without disturbing the peripheral mononuclear cell layer at the interface, transferred to a sterile $1.5-\mathrm{mL}$ centrifuge tube, labeled and stored at $-80^{\circ} \mathrm{C}$ and used later for these experiments. 


\section{Depletion of Acute Myeloid Leukemia Samples}

Crude plasma samples were depleted using Pierce ${ }^{\circledR}$ Albumin/IgG Removal Kit (according to manufacturer's instructions) prior to proteomics analysis in order to remove the most abundance proteins especially albumin and $\operatorname{IgG}$ and determination of total protein concentration was performed by Bradford technique. ${ }^{7}$

\section{Disease Progression}

In this experiment, 6 samples were analyzed in two arms. The first arm compared BM plasma and PB plasma at diagnosis to evaluate the differences in the number of proteins identified in each sample type and to observe the changes in expression between these two types of bodily fluid compartments specimens as baseline protein patterns. The second arm of the analysis was to evaluate changes in the expression of proteins in PB plasma at the time of diagnosis compared to protein changes at remission period.

\section{Risk Categories}

The comparison was made between different categorization of 6 AML leukemia samples including low-, intermediate- and high-risks in order to follow the differentiation of proteins expressions within these risks using PBP samples at time of diagnosis.

\section{Sample Preparation and Protein In-solution Digestion}

All samples were normalized for protein concentration. From each sample group, equal amount (100 $\mu \mathrm{g}$ ) of complex protein mixtures derived from depleted plasma was taken from disease progression and risk category and exchanged twice with 500 $\mu \mathrm{L}$ of $0.1 \%$ RapiGest (Waters, Manchester, UK) using a 3-kDa ultra filtration device (Millipore), previously described. ${ }^{8,9}$ Protein concentrations of 0.5 to $1 \mu \mathrm{g} / \mu \mathrm{L}$ were achieved at the end of in-solution tryptic digestion. Briefly, the proteins were denatured in $0.1 \%$ RapiGest $\mathrm{SF}$ at $80^{\circ} \mathrm{C}$ for $15 \mathrm{~min}$, reduced in $10 \mathrm{mM}$ DTT at $60^{\circ} \mathrm{C}$ for $30 \mathrm{~min}$, centrifuged briefly at $13,000 \mathrm{RPM}$ for 10 seconds to bring together the condensation under the tube cap, allowed to cool to room temperature, and alkylated in $10 \mathrm{mM}$ iodoacetamide (IAA) for $40 \mathrm{~min}$ at room temperature in the dark. The samples were then trypsin-digested at an enzyme: protein ratio $(\mathrm{w} / \mathrm{w}$; $1 \mu \mathrm{g} / \mu \mathrm{L}$ trypsin concentration) of 1:50 overnight at $37^{\circ} \mathrm{C}$ with gentle shaking. The digestion/ RapiGest was quenched by incubation with $4 \mu \mathrm{L}$ of $12 \mathrm{M}$ $\mathrm{HCl}$ at $37^{\circ} \mathrm{C}$ for $15 \mathrm{~min}$, followed by centrifugation at 13,000 RPM for $10 \mathrm{~min}$. The samples were then diluted to $5 \mathrm{pmol} / \mu \mathrm{L}$ or 5 - to 10 -fold with aqueous $0.1 \%$ formic acid to achieve a load of approximately $3 \mu \mathrm{g}$ for the analytical column of the mass spectrometer. All samples were spiked with yeast alcohol dehydrogenase (ADH; P00330) as an internal standard at a concentration of $200 \mathrm{fmol}$ per injection to facilitate absolute quantitation as previously described. ${ }^{10,11}$

\section{Protein Identification by Synapt G2 Mass Spectrometry}

A one-dimensional, (1-D) Nano Acquity liquid chromatography coupled with a Synapt G2 high definition tandem mass spectrometry (Waters, Manchester, UK) was used to generate expression proteomics data for three subtypes of AML samples. The optimization of the instrument was as previously described [10]. Briefly, the detector set up was done using 2-ng/ $\mu$ L-leucine enkephalin (556.277 Da). Mass/charge (m/z) calibration was achieved with a separate infusion of $500 \mathrm{fmol}$ [Glu] 1-Fibrinopeptide B (GluFib, 785.843 Da), on a Trizaic Infusion tile using the automation of the Mass Lynx IntelliStart. Other parameters that were preset were capillary voltage at $3.6 \mathrm{Kv}$, sample cone at $50 \mathrm{~V}$, extraction cone of $5 \mathrm{~V}$, source temperature at $100^{\circ} \mathrm{C}$, cone gas flow rate of $10 \mathrm{~L} / \mathrm{h}$, Nano-flow gas pressure at 0.6 bars, and a purge gas flow rate of $600 \mathrm{~L} / \mathrm{h}$. All analyses were performed on a Trizaic Nano source (Waters, Manchester, UK), using the positive ion mobility mode nanoESI at slow flow rate.

A $3 \mu \mathrm{l}$ sample containing approximately $3 \mu \mathrm{g}$ of the digested protein was loaded onto the column, and samples were infused using the Acquity Sample Manager with a mobile phase consisting of A1 (99\% water $+1 \%$ acetonitrile $+0.1 \%$ formic acid) and B1 $(100 \%$ acetonitrile $+0.1 \%$ formic acid, with a sample flow rate of $0.450 \mu 1 / \mathrm{min})$. Data-in- 


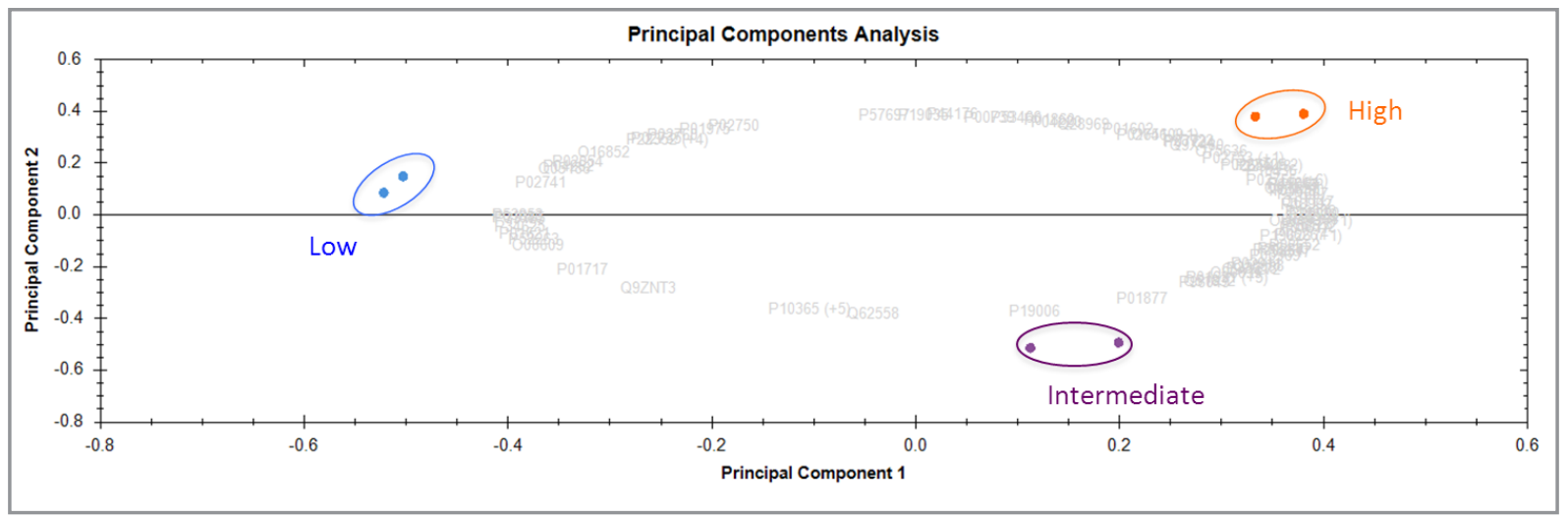

Figure 1. Unsupervised Principal Component Analysis (PCA) plot using the 145 proteins that were significantly differentially expressed between low- (purple), intermediate- (blue), and high-risk (orange) plasma samples form AML patients. The numbers in grey scale are the accession numbers of each of the proteins. The PCA plot was generated from Progenesis QI for proteomics using the normalized quantitative dataset of protein expression changes across the three sample sub-groups.

dependent acquisition/iron mobility separation experiments (MSEs) were performed, and data were acquired over an m/z range of 50 - $2000 \mathrm{Da}$, scan time of $1 \mathrm{sec}$, ramped transfer collision energy of 20 to $50 \mathrm{~V}$, and total acquisition time of $120 \mathrm{~min}$. All samples were run in triplicate and repeated in 2 different experiments to ensure reproducibility of results. The data were accessed via the Mass Lynx program (Version. 4.1, SCN833, Waters, Manchester, UK) using the resolution and positive-polarity modes. The data were background subtracted, smoothed, and de-isotoped at a medium threshold. Protein Lynx Global Server (PLGS) 2.2 (Waters, Manchester, UK) and Progenesis LC/MS (QI) were used for all automated data processing and database searches.

The generated peptide masses were compared against the SwissProt protein sequence database and cross-validated on Uniprot human proteome database using PLGS 2.5/Progenesis QI (Waters, UK, Nonlinear, UK) for protein identification and differential analysis as previously described.10, 11

\section{Data Analysis and Informatics}

Progenesis QI (Nonlinear Dynamics, Newcastle, UK) software was used for data processing and search. The search criteria were; 1 missed cleavage, max protein mass $=1000 \mathrm{kDa}$, trypsin, and Carbamidomethyl $\mathrm{C}$ fixed and oxidation $\mathrm{M}$ variable modifications. The software generated normalized label-free relative quantification analyses and significantly differentially polypeptides were subjected to unsupervised principal component analyses (PCA) for all sample groups.

Multivariate data analysis Variance (ANOVA) at $\mathrm{p} \leq 0.05$ was used to identify significant alterations in regulated proteins and in addition, the expression level of at least $>1.5$-fold change between paired of samples being compared.

\section{RESULTS}

\section{Differentially Expressed Proteins in Acute Myeloid Leukemia Patients at Diagnosis and Remission}

Among the limitations facing prospective sample collection for expression analysis is logistics in prospective serial collection of samples at different stages of disease progression. Hence it was possible to have either BMP or PBP and where possible both from the same individual patients at diagnosis. While in majority of cases, only PBP was available as at time of disease remission. Marked differentially expressed proteins were observed when all the three sample types including BMP and PBP at diagnosis as well as PBP at remission were subjected to non-gel-based and label-free insolution expression proteomics analyses. In total, 39 identified proteins were differentially expressed across all the three sample types. However, only 21 of the 39 proteins showed significant $(\mathrm{p}<0.05)$ expression changes and at least $\geq 1.5$-fold difference 


\begin{tabular}{|c|c|c|c|c|}
\hline Accession\# & $\begin{array}{l}\text { Max } \\
\text { Fold } \\
\text { Change }\end{array}$ & $\begin{array}{l}\text { Highest } \\
\text { Mean } \\
\text { Condition }\end{array}$ & $\begin{array}{l}\text { Lowest } \\
\text { Mean } \\
\text { Condition }\end{array}$ & Protein Description \\
\hline О83974 & 1.06 & Diagnosis & Remission & Nucleoside diphosphate kinase (EC 2.7.4.6) (NDK). \\
\hline P02048 & 2.60 & Diagnosis & Remission & Hemoglobin beta chain. \\
\hline P02774 & 2.26 & Remission & Diagnosis & $\begin{array}{l}\text { Vitamin D-binding protein precursor (DBP) } \\
\text { (Group-specific component). }\end{array}$ \\
\hline P01617 & 2.02 & Diagnosis & Remission & Ig kappa chain V-II region TEW. \\
\hline P01623 & 1.35 & Remission & Diagnosis & Ig kappa chain V-III region WOL. \\
\hline P02735 & 3.70 & Diagnosis & Remission & $\begin{array}{l}\text { Serum amyloid A protein precursor (SAA) [Contains: } \\
\text { Amyloid protein A SAA1,SAA2 ]. }\end{array}$ \\
\hline P04217 & 2.16 & Remission & Diagnosis & Alpha-1B-glycoprotein. \\
\hline P80748 & 1.82 & Remission & Diagnosis & Ig lambda chain V-III region LOI. \\
\hline Q60397 & 5.81 & Diagnosis & Remission & Guanine nucleotide-binding protein $G(\mathbf{k})$, alpha subunit. \\
\hline P01766 & 1.73 & Diagnosis & Remission & Ig heavy chain V-III region BRO. \\
\hline P01922 & 4.62 & Diagnosis & Remission & Hemoglobin alpha chain. \\
\hline P00738 & 1.86 & Diagnosis & Remission & Haptoglobin-2 precursor. \\
\hline P01009 & 1.53 & Diagnosis & Remission & Alpha-1-antitrypsin precursor (Alpha-1 protease inhibitor). \\
\hline P01876 & 1.68 & Diagnosis & Remission & Ig alpha-1 chain $\mathrm{C}$ region. \\
\hline Q28522 & 1.03 & Diagnosis & Remission & Serum albumin precursor (Fragment). \\
\hline P02790 & 1.63 & Remission & Diagnosis & Hemopexin precursor (Beta-1B-glycoprotein). \\
\hline P01871 & 2.49 & Diagnosis & Remission & Ig $\mathrm{MU}$ chain $\mathrm{C}$ region. \\
\hline P02769 & 1.88 & Remission & Diagnosis & Serum albumin precursor (Allergen Bos d 6). \\
\hline P01860 & 1.32 & Diagnosis & Remission & Ig gamma-3 chain $\mathrm{C}$ region (Heavy chain disease protein). \\
\hline
\end{tabular}

between PB plasma samples obtained at diagnosis and at remission from the same individual patients. Three of the 21 proteins with expression difference of greater than 3-fold were listed in (Table 1).

\section{Differentially Expressed Plasma Proteins Be- tween Different Risk Categories of Acute My- eloid Leukemia}

Approximately 225-250 unique proteins species were identified from each of triplicate runs from all sample groups including low-, intermediate-, and high-risk patients. Among these proteins, 145 were significantly differentially expressed with at least $\geq 1.5$-fold change $(\mathrm{p}<0.05)$. The dataset of 145 differentially expressed proteins was subjected to unsupervised Principal Component Analysis (PCA) and samples were distinctively clustered into three separate groups (Figure 1). The majority of the differentially expressed proteins were up-regulated among the high AML-risk category, followed in decreasing order of expression of the proteins in the intermediate- and low risk categories respectively as listed in Table 2.

\section{DISCUSSION}

Despite advanced improvement in the diagnosis and management of hematological diseases including AML, accurate prognostication of disease often remain elusive using currently available clinical and cytogenetic parameters. The heterogeneity of AML is detectable via morphological and immunological evaluation as well as through their cytogenetic and molecular characteristics. These cellular characteristics are defined by the molecular entities, which could be targeted for the development of diagnostic and prognostic profiles, and for the identification of disease subtypes. ${ }^{12}$ Despite the currently available therapeutic methods, one of which is stem cell transplantation, the rate of recovery for patients with AML is still rather low. While long-term survival is high in older adults with AML, the majority of patients are incapable of tolerating chemotherapy. Even though the patholo- 
International Journal of Hematology and Oncology

\begin{tabular}{|c|c|c|c|c|c|c|c|}
\hline Accession & $\begin{array}{l}\text { Peptide } \\
\text { count }\end{array}$ & $\begin{array}{l}\text { Peptides } \\
\text { used for } \\
\text { Quant }\end{array}$ & Anova (p) & $\begin{array}{l}\text { Max fold } \\
\text { change }\end{array}$ & $\begin{array}{l}\text { Highest } \\
\text { mean } \\
\text { condition }\end{array}$ & $\begin{array}{l}\text { Lowest } \\
\text { mean } \\
\text { condition }\end{array}$ & Description \\
\hline Q29443 & 18 & 3 & $1.87 \mathrm{E}-05$ & 10.0016 & High & Low & $\begin{array}{l}\text { Serotransferrin precursor (Siderophilin) } \\
\text { (Beta-1-metal-binding globulin). }\end{array}$ \\
\hline P02753 & 4 & 3 & 3.60E-05 & 3.012 & High & Low & Plasma retinol-binding protein precursor (PRBP) (RBP). \\
\hline O74109 & 2 & 2 & 4.55E-05 & 3.851 & High & Intermediate & Acidic ribosomal protein PO homolog (L10E). \\
\hline Q9WZ72 & 1 & 1 & $5.65 \mathrm{E}-05$ & 6.410 & Intermediate & Low & $30 \mathrm{~S}$ ribosomal protein S6. \\
\hline P44176 & 1 & 1 & 0.000114372 & 3.166 & High & Intermediate & Protein trpH. \\
\hline P01880 & 6 & 6 & 0.000119906 & 26.529 & Low & High & Ig delta chain $\mathrm{C}$ region. \\
\hline P02741 & 8 & 8 & 0.000189273 & 2.834 & Low & Intermediate & C-reactive protein precursor. \\
\hline P19006 & 13 & 1 & 0.000204301 & Infinity & Intermediate & Low & Haptoglobin alpha and beta chains. \\
\hline P02911 & 3 & 3 & 0.014019105 & 1854.053 & Intermediate & Low & Lysine-arginine-ornithine-binding periplasmic protein. \\
\hline P15514 & 1 & 1 & 0.033543696 & 112.135 & Low & Intermediate & Amphiregulin precursor (AR) (Colorectum cell-derived). \\
\hline P36421 & 1 & 1 & 0.04183228 & Infinity & Intermediate & High & Tyrosyl-tRNA synthetase, cytoplasmic (EC 6.1.1.1). \\
\hline P23722 & 3 & 2 & 0.000249133 & 2.332 & High & Low & Glyceraldehyde 3-phosphate dehydrogenase (EC 1.2.13). \\
\hline P02760 & 10 & 10 & 0.000252698 & 2.109 & Low & Intermediate & $\begin{array}{l}\text { AMBP protein precursor [Contains: } \\
\text { Alpha-1-microglobulin]. }\end{array}$ \\
\hline P01602 & 2 & 2 & 0.000262566 & 7.169 & High & Intermediate & Ig kappa chain V-I region HK102 precursor. \\
\hline P02652 & 7 & 7 & 0.000294418 & 3.199 & Intermediate & Low & Apolipoprotein A-II precursor (Apo-All) (ApoA-II). \\
\hline P04262 & 1 & 1 & 0.000314701 & 2.313 & Low & Intermediate & Keratin, type II cytoskeletal 68 kDa, component IB. \\
\hline P21667 & 5 & 2 & 0.000325455 & 39.825 & High & Low & Hemoglobin beta chain. \\
\hline P01871 & 23 & 9 & 0.000384945 & 1.963 & High & Intermediate & Ig MU chain $\mathrm{C}$ region. \\
\hline P02765 & 15 & 14 & 0.000386585 & 2.132 & High & Low & $\begin{array}{l}\text { Alpha-2-HS-glycoprotein precursor (Fetuin-A) } \\
\text { (Alph-2-HS-glycoprotein). }\end{array}$ \\
\hline 008609 & 2 & 1 & 0.00040561 & 6.050 & Low & High & Transcription factor-like protein 4 (Max-like bHLHZip protein). \\
\hline O16852 & 2 & 2 & 0.000491965 & 2.854 & Low & Intermediate & Proliferating cell nuclear antigen (PCNA) (Cyclin). \\
\hline P01860 & 12 & 5 & 0.000513446 & 5.569 & High & Intermediate & Ig gamma-3 chain $\mathrm{C}$ region (Heavy chain disease protein). \\
\hline P53853 & 1 & 1 & 0.000531913 & 8.174 & Low & High & Hypothetical 30.6 kDa protein in RPA49-SUl1 integrity. \\
\hline P52263 & 1 & 1 & 0.000546844 & 2.622 & Low & High & Ovomucoid (Fragment). \\
\hline P02054 & 3 & 1 & 0.000560865 & 2.725 & Low & Intermediate & Hemoglobin beta chain. \\
\hline P54398 & 3 & 2 & 0.00062032 & 2.758 & High & Low & Fat body protein P6. \\
\hline P02647 & 28 & 21 & 0.000644801 & 1.892 & Intermediate & Low & Apolipoprotein A-I precursor (Apo-Al). \\
\hline O75636 & 7 & 7 & 0.000655277 & 2.602 & High & Low & $\begin{array}{l}\text { Ficolin } 3 \text { precursor (Collagen/fibrinogen } \\
\text { domain-containing protein 3). }\end{array}$ \\
\hline P02655 & 2 & 2 & 0.00094464 & 7.785 & High & Low & Apolipoprotein C-II precursor (Apo-CII). \\
\hline P016319 & 7 & 3 & 0.000985358 & 8.544 & Intermediate & Low & Ig kappa chain V-II region 26-10. \\
\hline P05090 & 7 & 7 & 0.000985493 & 2.620 & High & Low & Apolipoprotein D precursor (ApoD). \\
\hline P34625 & 1 & 1 & 0.00113035 & 27.706 & Low & High & $\begin{array}{l}\text { Hypothetical } 10.6 \text { kDa protein ZK353.2 in } \\
\text { chromosome III. }\end{array}$ \\
\hline P04208 & 4 & 1 & 0.001164622 & 1.842 & Intermediate & High & Ig lambda chain V-I region WAH. \\
\hline P01877 & 20 & 3 & 0.001298204 & 2.070 & Intermediate & Low & Ig alpha-2 chain $\mathrm{C}$ region. \\
\hline P02768 & 77 & 32 & 0.001319554 & 1.949 & High & Intermediate & Serum albumin precursor. \\
\hline P19652 & 10 & 9 & 0.001334186 & 1.680 & High & Intermediate & Alpha-1-acid glycoprotein 2 precursor (AGP 2). \\
\hline P29621 & 4 & 2 & 0.001350336 & 1.846 & Intermediate & Low & Kallikrein-binding protein precursor (KBP). \\
\hline P02750 & 15 & 15 & 0.001458032 & 2.490 & Low & Intermediate & Leucine-rich alpha-2-glycoprotein (LRG). \\
\hline P25311 & 15 & 15 & 0.001486352 & 1.796 & High & Intermediate & $\begin{array}{l}\text { Zinc-alpha-2-glycoprotein precursor } \\
\text { (Zn-alpha-2-glycoprotein). }\end{array}$ \\
\hline P02735 & 5 & 5 & 0.001487066 & 3.804 & Low & Intermediate & $\begin{array}{l}\text { Serum amyloid A protein precursor (SAA) } \\
\text { [Contains: Amyloid protein A]. }\end{array}$ \\
\hline P02656 & 4 & 2 & 0.001548529 & 4.508 & High & Intermediate & Apolipoprotein C-III precursor (Apo-CIII). \\
\hline P01975 & 4 & 2 & 0.00166841 & 2.165 & Low & Intermediate & Hemoglobin alpha chain. \\
\hline P50512 & 2 & 2 & 0.001838578 & 4.443 & High & Low & RNA polymerase sigma-32 factor. \\
\hline Q58998 & 1 & 1 & 0.002023546 & 6.297 & Intermediate & Low & Probable ribose 5-phosphate isomerase (EC 5.3.1.6) \\
\hline Q00137 & 2 & 2 & 0.002121304 & 1.919 & Low & Intermediate & Hypothetical gene 8 membrane protein. \\
\hline P58517 & 1 & 1 & 0.002168685 & 6.652 & Intermediate & Low & Serine protease inhibitor 4 (PI-4) (Fragment). \\
\hline P01717 & 2 & 1 & 0.002612881 & 12.074 & Low & High & Ig lambda chain V-IV region Hil. \\
\hline
\end{tabular}


International Journal of Hematology and Oncology

\begin{tabular}{|c|c|c|c|c|c|c|c|}
\hline Accession & $\begin{array}{l}\text { Peptide } \\
\text { count }\end{array}$ & $\begin{array}{l}\text { Peptides } \\
\text { used for } \\
\text { Quant }\end{array}$ & $\begin{array}{l}\text { Anova (p) } \\
\text { change }\end{array}$ & $\begin{array}{l}\text { Max fold } \\
\text { mean } \\
\text { condition }\end{array}$ & $\begin{array}{l}\text { Highest } \\
\text { mean } \\
\text { condition }\end{array}$ & Lowest & Description \\
\hline P98049 & 1 & 1 & 0.002986535 & 5.594 & Intermediate & Low & Cytochrome c oxidase polypeptide II (EC 1.9.3.1). \\
\hline P53466 & 3 & 1 & 0.003026519 & 2.524 & High & Intermediate & Actin, cytoskeletal 2 (LPC2). \\
\hline P01630 & 1 & 1 & 0.003039135 & 3.532 & High & Low & Ig kappa chain V-II region 7S34.1. \\
\hline P07490 & 2 & 1 & 0.003137362 & 2.866 & High & Low & Progonadoliberin I precursor [Contains: Gonadoliberin II]. \\
\hline P01621 & 2 & 1 & 0.003272698 & 5.787 & Low & High & Ig kappa chain V-III region NG9 precursor (Fragment). \\
\hline P01857 & 15 & 5 & 0.003351987 & 1.907 & High & Low & Ig gamma-1 chain $\mathrm{C}$ region. \\
\hline P01011 & 17 & 15 & 0.003405063 & 1.651 & Low & Intermediate & Alpha-1-antichymotrypsin precursor (ACT). \\
\hline P31227 & 3 & 3 & 0.003424816 & 1.500 & Low & High & S-modulin (Sensitivity-modulating protein). \\
\hline P09871 & 10 & 10 & 0.003514922 & 1.547 & High & Low & Complement C1s component precursor (EC 3.4.21.42) \\
\hline P49064 & 15 & 4 & 0.003568207 & 2.425 & High & Low & Serum albumin precursor (Allergen Fel d 2). \\
\hline P01862 & 5 & 2 & 0.003616366 & 1.771 & Low & Intermediate & Ig gamma-2 chain $\mathrm{C}$ region. \\
\hline Q9X480 & 2 & 2 & 0.003655911 & 2.118 & High & Low & Putative signal peptide peptidase sppA (EC 3.4.21). \\
\hline P29610 & 3 & 3 & 0.003661547 & 1.550 & High & Low & Cytochrome c1, heme protein, mitochondrial precursor. \\
\hline Q28969 & 1 & 1 & 0.00375721 & 4.033 & High & Intermediate & Nitric-oxide synthase, endothelial (EC 1.14.13.39). \\
\hline P06727 & 23 & 23 & 0.003832552 & 1.856 & High & Low & Apolipoprotein A-IV precursor (Apo-AIV). \\
\hline Q09163 & 4 & 3 & 0.004158717 & 1.827 & Low & High & Delta-like protein precursor (DLK) (Preadipocyte factor 1). \\
\hline Q00835 & 1 & 1 & 0.004316318 & 8.127 & Intermediate & Low & Trichodiene synthase (EC 4.2.3.6) (Sesquiterpene cyclase). \\
\hline Q28522 & 35 & 3 & 0.004421518 & 7.094 & High & Low & Serum albumin precursor (Fragment). \\
\hline P27425 & 13 & 2 & 0.004543771 & 4.626 & High & Low & $\begin{array}{l}\text { Serotransferrin precursor (Siderophilin) } \\
\text { (Beta-1-metal binding globulin). }\end{array}$ \\
\hline P18436 & 2 & 1 & 0.004599351 & 2.107 & High & Low & Hemoglobin gamma chain. \\
\hline P04090 & 2 & 2 & 0.005033086 & 1.677 & Low & High & Prorelaxin $\mathrm{H} 2$ precursor. \\
\hline P00738 & 42 & 16 & 0.005072056 & 1.560 & Low & High & Haptoglobin-2 precursor. \\
\hline 060493 & 2 & 2 & 0.00508749 & 7.187 & High & Low & Sorting nexin 3 (SDP3 protein). \\
\hline P56410 & 11 & 2 & 0.005121536 & 2.622 & Intermediate & Low & Ovotransferrin. \\
\hline P50450 & 5 & 1 & 0.005482226 & 1.834 & Low & High & Thyroxine-binding globulin precursor (T4-binding globulin). \\
\hline P00969 & 2 & 2 & 0.005512066 & 8.731 & Intermediate & Low & $\begin{array}{l}\text { DNA ligase (EC 6.5.1.1) (Polydeoxyribonucleotide } \\
\text { synthase). }\end{array}$ \\
\hline Q05186 & 1 & 1 & 0.005570395 & 4.633 & Low & Intermediate & Reticulocalbin 1 precursor. \\
\hline P07724 & 10 & 1 & 0.005644092 & 2.050 & High & Low & Serum albumin precursor. \\
\hline P33213 & 5 & 5 & 0.005692555 & 2.075 & Intermediate & Low & Nodulation protein nolX. \\
\hline P18902 & 2 & 1 & 0.005852563 & 3.176 & High & Low & Plasma retinol-binding protein (PRBP) (RBP). \\
\hline P01884 & 2 & 1 & 0.005950654 & 1.825 & Low & Intermediate & Beta-2-microglobulin precursor. \\
\hline P39187 & 1 & 1 & 0.006075748 & 1.736 & Low & High & Protein ytfJ precursor. \\
\hline P02790 & 21 & 19 & 0.006116569 & 1.854 & High & Intermediate & Hemopexin precursor (Beta-1B-glycoprotein). \\
\hline Q62558 & 17 & 4 & 0.006132337 & 2.236 & Intermediate & High & Haptoglobin precursor. \\
\hline P19002 & 7 & 1 & 0.006135569 & 2.669 & High & Low & Hemoglobin alpha-1, alpha-2, and alpha-3 chains. \\
\hline P06123 & 1 & 1 & 0.006432199 & 7.061 & High & Low & Ferredoxin-like protein in vnf region. \\
\hline P57697 & 1 & 1 & 0.00649747 & 5.219 & High & Intermediate & Proteasome alpha subunit (EC 3.4.25.1) (Multicatal). \\
\hline Q51832 & 2 & 2 & 0.007133136 & 2.543 & Intermediate & Low & Ribonuclease HII (EC 3.1.26.4) (RNase HIII) (Fragment). \\
\hline P33165 & 2 & 1 & 0.007464601 & 3.651 & Low & High & Elongation factor Tu (EF-Tu). \\
\hline Q61147 & 16 & 4 & 0.007730386 & 3.483 & High & Low & Ceruloplasmin precursor (EC 1.16.3.1) (Ferroxidase). \\
\hline P04170 & 2 & 2 & 0.007834218 & 1.703 & Intermediate & High & Rubredoxin (Rd). \\
\hline P19626 & 1 & 1 & 0.008320912 & 2.348 & Intermediate & Low & Myosin regulatory light chain 2. \\
\hline P19035 & 3 & 2 & 0.008619178 & 4.930 & High & Intermediate & Apolipoprotein C-III (Apo-CIII) (Fragment). \\
\hline Q9PRL9 & 4 & 2 & 0.008649686 & 1.624 & High & Low & Hemoglobin alpha-I chain. \\
\hline Q51929 & 1 & 1 & 0.009014932 & 1.860 & Low & Intermediate & Major cold-shock protein (Fragment). \\
\hline Q9ZE57 & 1 & 1 & 0.009220375 & 2.281 & High & Low & Hypothetical protein RP090. \\
\hline P01763 & 3 & 2 & 0.009823443 & 1.885 & Low & High & Ig heavy chain V-III region WEA. \\
\hline P01781 & 4 & 2 & 0.010223347 & 1.511 & Low & Intermediate & Ig heavy chain V-III region GAL. \\
\hline P51884 & 8 & 6 & 0.010322692 & 1.755 & High & Low & $\begin{array}{l}\text { Lumican precursor (LUM) (Keratan sulfate } \\
\text { proteoglycan lumican). }\end{array}$ \\
\hline P09904 & 4 & 1 & 0.011026794 & 1.678 & High & Low & Hemoglobin alpha chain. \\
\hline P01743 & 2 & 2 & 0.011393916 & 4.619 & High & Low & Ig heavy chain V-I region HG3 precursor. \\
\hline
\end{tabular}


International Journal of Hematology and Oncology

\begin{tabular}{|c|c|c|c|c|c|c|c|}
\hline Accession & $\begin{array}{l}\text { Peptide } \\
\text { count }\end{array}$ & $\begin{array}{l}\text { Peptides } \\
\text { used for } \\
\text { Quant }\end{array}$ & Anova (p) & $\begin{array}{l}\text { Max fold } \\
\text { change }\end{array}$ & $\begin{array}{l}\text { Highest } \\
\text { mean } \\
\text { condition }\end{array}$ & $\begin{array}{l}\text { Lowest } \\
\text { mean } \\
\text { condition }\end{array}$ & Description \\
\hline P10365 & 3 & 3 & 0.011657067 & 3.012 & Intermediate & High & Actin. \\
\hline P04442 & 4 & 2 & 0.011928285 & 1.581 & Intermediate & Low & Hemoglobin alpha-D chain. \\
\hline Q9P4C2 & 7 & 1 & 0.012101487 & 1.763 & Intermediate & Low & Alcohol dehydrogenase II (EC 1.1.1.1). \\
\hline P01617 & 2 & 2 & 0.012223837 & 2.319 & High & Low & Ig kappa chain V-II region TEW. \\
\hline Q9ZNT3 & 1 & 1 & 0.012614214 & 87.409 & Low & High & Actin-depolymerizing factor 5 (ADF-5) (AtADF5). \\
\hline P06267 & 1 & 1 & 0.012872182 & 2.268 & High & Low & $\begin{array}{l}\text { Light-independent protochlorophyllide reductase } \\
\text { iron-sulfur-ATP-binding protein. }\end{array}$ \\
\hline P20284 & 2 & 1 & 0.012912974 & 7.010 & Intermediate & Low & 30 S ribosomal protein S19P (HmaS19) (HS18). \\
\hline P00739 & 32 & 8 & 0.013284412 & 2.228 & High & Intermediate & Haptoglobin-related protein precursor. \\
\hline P27169 & 9 & 9 & 0.013389271 & 1.736 & High & Intermediate & Serum paraoxonase/arylesterase 1 (EC 3.1.1.2) (EC 3.1.8.1). \\
\hline P22352 & 4 & 4 & 0.014523229 & 2.117 & Low & Intermediate & Plasma glutathione peroxidase precursor (EC 1.11.1.9) \\
\hline P04220 & 15 & 1 & 0.015439708 & 3.003 & High & Intermediate & Ig MU heavy chain disease protein (BOT). \\
\hline P46755 & 4 & 3 & 0.017212861 & 1.522 & Low & Intermediate & Mitochondrial ribosomal protein S4. \\
\hline P01876 & 22 & 2 & 0.017265181 & 1.786 & Intermediate & Low & Ig alpha-1 chain $\mathrm{C}$ region. \\
\hline P41263 & 3 & 3 & 0.017476193 & 1.683 & High & Low & Plasma retinol-binding protein precursor (PRBP). \\
\hline P73836 & 2 & 2 & 0.017971459 & 1.709 & Intermediate & Low & Hypothetical $10.7 \mathrm{kDa}$ protein ssr3402 precursor. \\
\hline Q03044 & 2 & 1 & 0.018080499 & 2.041 & Low & Intermediate & Alpha-1-antiproteinase precursor (Alpha-1-antitrypsin) \\
\hline P02788 & 8 & 2 & 0.020697749 & 2.304 & Intermediate & Low & $\begin{array}{l}\text { Lactotransferrin precursor (Lactoferrin) [Contains; } \\
\text { Lactoferroxin A, B and C]. }\end{array}$ \\
\hline P07461 & 1 & 1 & 0.021366279 & 3.514 & Intermediate & Low & Myosin regulatory light chain, smooth muscle. \\
\hline P08835 & 9 & 1 & 0.021598078 & 6.494 & Low & High & Serum albumin precursor (Fragment). \\
\hline P05543 & 11 & 6 & 0.022442445 & 1.814 & Low & Intermediate & Thyroxine-binding globulin precursor (T4-binding globulin). \\
\hline P00915 & 2 & 1 & 0.023901829 & 4.762 & High & Low & $\begin{array}{l}\text { Carbonic anhydrase I (EC 4.2.1.1) (Carbonate } \\
\text { dehydrogenase). }\end{array}$ \\
\hline P43067 & 3 & 1 & 0.024431418 & 7.063 & High & Low & Alcohol dehydrogenase 1 (EC 1.1.1.1) (40 kDa allergen). \\
\hline P01610 & 2 & 1 & 0.024732175 & 8.178 & Intermediate & Low & Ig kappa chain V-I region WEA. \\
\hline Q04791 & 3 & 3 & 0.025244992 & 2.486 & Intermediate & Low & $\begin{array}{l}\text { Fatty acyl-CoA hydrolase precursor, medium chain } \\
\text { (EC: } 3.1 .2 . \text {-). }\end{array}$ \\
\hline P38792 & 2 & 1 & 0.026964975 & 1.808 & Low & Intermediate & Exosome complex exonuclease RRP4 (EC 3.1.13.-) (Ri). \\
\hline P28030 & 1 & 1 & 0.029020189 & 2.701 & Low & High & Thermostable direct hemolysin precursor. \\
\hline P50828 & 3 & 2 & 0.029509667 & 6.195 & High & Low & Hemopexin precursor (Hyaluronidase) (EC 3.2.1.35). \\
\hline P02023 & 22 & 2 & 0.029551727 & 1.848 & High & Low & Hemoglobin beta chain. \\
\hline P21380 & 8 & 1 & 0.03028008 & 1.989 & Intermediate & Low & Hemoglobin beta chain. \\
\hline P00488 & 9 & 9 & 0.030287679 & 1.597 & High & Low & Coagulation factor XIII A chain precursor (EC 2.3.2.13). \\
\hline P00331 & 6 & 2 & 0.030341899 & 2.771 & High & Low & Alcohol dehydrogenase II (EC 1.1.1.1). \\
\hline P02746 & 3 & 2 & 0.030684442 & 3.279 & Low & Intermediate & Complement $\mathrm{C} 1 \mathrm{q}$ subcomponent, $\mathrm{B}$ chain precursor. \\
\hline P35217 & 2 & 1 & 0.032376447 & 21.464 & High & Low & $\begin{array}{l}\text { Carbonic anhydrase I (EC 4.2.1.1) (Carbonate } \\
\text { dehydrogenase). }\end{array}$ \\
\hline P01600 & 2 & 1 & 0.032645748 & 1.943 & High & Intermediate & Ig kappa chain V-I region Hau. \\
\hline P01594 & 2 & 1 & 0.03295854 & 4.125 & High & Intermediate & Ig kappa chain V-I region AU. \\
\hline P01024 & 40 & 38 & 0.033275096 & 1.552 & High & Intermediate & Complement C3 precursor [Contains: C3a anaphylatoxin]. \\
\hline P09235 & 1 & 1 & 0.033829635 & 1.553 & Low & Intermediate & Interferon alpha-9 precursor. \\
\hline P00761 & 6 & 5 & 0.03447314 & 1.775 & Low & Intermediate & Trypsin precursor (EC 3.4.21.4). \\
\hline P01966 & 8 & 1 & 0.037099364 & 16.199 & Intermediate & Low & Hemoglobin alpha chain. \\
\hline P36957 & 1 & 1 & 0.037248952 & 3.939 & Intermediate & High & $\begin{array}{l}\text { Dihydrolipoamide succinyltransferase component of } \\
\text { 2-oxoglutarate dehydrogenase complex. }\end{array}$ \\
\hline P05611 & 1 & 1 & 0.03741116 & 2.102 & High & Low & Ovomucoid (Fragment). \\
\hline Q28932 & 7 & 1 & 0.038642909 & 2.839 & High & Low & Hemoglobin beta chain. \\
\hline P81398 & 4 & 4 & 0.039208009 & 1.737 & High & Low & Rhodocetin beta subunit. \\
\hline Q09886 & 1 & 1 & 0.039832596 & 2.635 & High & Low & Hypothetical 13.7 kDa protein C584.12 in chromosome III. \\
\hline O74258 & 4 & 2 & 0.040479378 & 1.844 & Intermediate & High & Actin. \\
\hline P07414 & 6 & 1 & 0.043441192 & 3.278 & High & Low & Hemoglobin alpha chain. \\
\hline P42538 & 4 & 2 & 0.044487833 & 1.810 & Intermediate & Low & Hypothetical 37.2 kDa protein (ORF3). \\
\hline
\end{tabular}


gy and the potential targets of AML have been well studied thus far, the disease sometimes cannot be effectively treated using standard pharmacological treatment techniques. ${ }^{13}$

This current study was has attempted to identify novel proteins that could be used as biomarkers for diagnosis, prognosis and mode of treatment of AML. The discovery of potential biomarkers for AML was explored via proteomic profiling of samples from AML patients at diagnosis and at disease remission and with special focus on different categorization of AML including low, intermediate, and high-risk groups. This type of analysis might offer a more objective way to stratified AML patients into different risk categories based on their unique protein fingerprints. Our evaluation of protein patterns between BM and PB were very similar implying that molecular changes in BM of AML patients could be well studied using peripheral blood samples that offers less degree of invasiveness compared with BM biopsy and or aspiration.

Marked quantitative changes were observed when PB plasma at diagnosis and at remission from the same individual subjects was compared. Among the 21 identified differentially expressed proteins, three of the 21 proteins with expression difference of greater than 3-fold were further evaluated for their role in the progression and response to achieving remission of the malignant cell clones. One of them is Guanine nucleotide-binding protein $\mathrm{G}(\mathrm{k}), \alpha$-subunit, which belongs to the family of $\mathrm{G}$ proteins. Some of the members of $\mathrm{G}$ proteins are known to function as intracellular molecular switches as well as their involvement in different signal transduction across the cell membrane. ${ }^{14}$

Of interest to the study is our observation of more than 5-fold difference in the expression of the Guanine nucleotide-binding protein $G(k), \alpha$-subunit between samples at diagnosis and remission.

Another protein with significantly up-regulated between samples at diagnosis and remission period is Serum amyloid A protein precursor (SAA; containing both amyloid precursor proteins SAA1 and SAA2). Increase plasma concentrations of these proteins are associated with changes during the acute phase response. While the proteins have been described to produce in the liver by the hepatocytes, however, extra hepatic productions have also be reported. ${ }^{15}$ Differential expression of serum amyloid A protein precursor has also been described also been reported in pediatric AML plasma samples. ${ }^{16}$

The fact that some of the identified proteins in this present study consisting of mainly samples from Adult AML patients might be indicative of similarity in the disease progression between pediatrics and adult AML patients.

Putting all these together signifies the potential roles of these molecules for prognostication of remission status of AML patients at diagnosis thus leading to the so-called personalized medicine.

In addition, it might be considered as a form of validation of potential usefulness of some of these proteins that shares some similarity in some of the differentially express proteins between adult and pediatrics AML subjects.

Different risk categories in AML including low-, intermediate-, and high-risk groups are being associated with probability of their success or failure to respond to therapy. We have identified some proteins that are significantly differentially expressed. In addition to quantitative changes in the levels of expression of some proteins, we have observed unique qualitative changes in four proteins that are either present in one of the three and absent in the other two, or in contrast, differentially expressed in two categories and absent in the third category.

Amphiregulin is a member of the epidermal growth factor family and with ability to promote the growth of normal epithelial cells as well its ability to restrains the growth of some malignant cells. ${ }^{17}$ Recently, Amphiregulin has been implicated with association human ovarian cancer progression. ${ }^{18,19}$ In this present study, we found Amphiregulin precursor (colorectum cell-derived growth factor) to be expressed in low risk AML samples and absent in both intermediate and high risk groups. While Amphiregulin role has not been extensively studied among hematological malignancy, its observed expression in this study is similar to changes in the plasma and bone marrow analyzed samples among low and high-risk pediatrics AML patients supporting its potential usefulness in risk stratification of both pediatrics as well as adult AML patients and 
they may contribute to better our understanding of the adverse heterogeneity of the disease at protein level.

\section{Conclusion}

This study has demonstrated application of expression proteomics towards discovery of novel biomarkers for objective stratification of AML risk categories as well as potential new targets for signature-based therapies of AML and for monitoring disease outcome.

\section{Acknowledgements}

The authors acknowledged administrative supports of the Research center Training and Education (RCTEO), the assistant and supports of Mr. Faisal Al Otaibi and his team at the logistics and purchasing department, $R C$ and the Research Center Administration at the King Faisal specialist hospital and research center, Saudi Arabia for their supports and provision of resources for this study.

\section{REFERENCES}

1. Luczak M, Kazmierczak M, Handschuh L, et al. Comparative proteome analysis of acute myeloid leukemia with and without maturation. J Proteomics 75: 5734-5748, 2012.

2. Boyd RS, Dyer MJ, Cain K. Proteomic analysis of b-cell malignancies. J Proteomics 73: 1804-1822, 2010.

3. Cui JW, Wang J, He K, et al. Proteomic analysis of human acute leukemia cells: insight into their classification. Clin Cancer Res 10: 6887-6896, 2004.

4. Monteoliva L, Albar JP. Differential proteomics: an overview of gel and non-gel based approaches. Brief Funct Genomic Proteomic 3: 220-239, 2004.

5. Quintás-Cardama A, Zhang N, Qui YH, et al. Loss of TRIM62 Expression Is an Independent Adverse Prognostic Factor in Acute Myeloid Leukemia. Clin Lymphoma Myeloma Leuk 14: 401-410, 2014.

6. Martorella A, Robbins R. Serum peptide profiling: identifying novel cancer biomarkers for early disease detection. Acta Biomedica 78: 123-128, 2007.

7. Bradford MM. A rapid and sensitive method for the quantitation of microgram quantities of protein utilizing the principle of protein-dye binding. Anal Biochem 72: 248-54, 1976.
8. Inutan E, Trimpin S. Laserspray ionization (LSI) ion mobility spectrometry (IMS) mass spectrometry. J Am Soc Mass Spectrom 21: 1260-1264, 2010.

9. Holcapek M, Jirásko R, Lísa M. Recent developments in liquid chromatography-mass spectrometry and related techniques. J Chromatogr A 1259: 3-15, 2012. doi: 10.1016/j. chroma.2012.08.072.

10. Alaiya A, Fox J, Bobis S, et al. Proteomic analysis of soft tissue tumor implants treated with a novel polybisphosphonate. Cancer Genomics Proteomics 11: 39-49, 2014.

11. Al-Moghrabi N, Nofel A, Al-Yousef N, et al. The molecular significance of methylated BRCA1 promoter in white blood cells of cancer-free females. BMC Cancer 14: 830, 2014.

12. Bacher U, Kohlmann A, Haferlach C, Haferlach T. Gene expression profiling in acute myeloid leukaemia (AML). Best Pract Res Clin Hematol 22: 169-180, 2009.

13. Hahn CK, Berchuck JE, Ross KN, et al. Proteomic and genetic approaches identify Syk as an AML target. Cancer Cell 16: 281-294, 2009

14. Andrew JM, Craig CM. Physiological Regulation of G ProteinLinked Signaling. Physiol Rev 79: 1373-1430, 1999.

15. Upragarin N, Landman WJM, Gaastra WE. Gruys Extra hepatic production of acute phase serum amyloid A. Histol Histopatho 20: 1295-1307, 2005.

16. Braoudaki M, Lambrou, Gl, Vougas K, et al. Protein biomarkers distinguish between high-and low-risk pediatric acute lymphoblastic leukemia in a tissue specific manner. $\mathrm{J}$ Hematol Oncol 6: 52, 2013.

17. Berasain C, Avila MA. Amphiregulin. Semin Cell Dev Biol 28 31-41, 2014.

18. So $W K$, Fan $Q$, Lau $M T$, et al. Amphiregulin induces human ovarian cancer cell invasion by down-regulating E-cadherin expression. FEBS letters 588: 3998-4007, 2014.

19. Cheng JC1, Chang HM, Leung PC. Epidermal growth factor induces human oviductal epithelial cell invasion by downregulating E-cadherin expression. J Clin Endocrinol Metab 97: 1380-1389, 2012.

\section{Correspondence}

Dr. Amer ALMAIMAN

Universiti Putra Malaysia, Institute of Biosciences

43400 UPM Serdang

Selangor, MALAYSIA

Tel: 0060172084424

Fax: 0060389472101

E-mail: ameralmeman@hotmail.com 